\title{
Precise Control of Highly Ordered Arrays of Nested Semiconductor/Metal Nanotubes
}

\author{
Diefeng $\mathrm{Gu}^{1,2}(\bowtie)$, , Helmut Baumgart ${ }^{1,2}$, Kandabara Tapily ${ }^{1,2}$, Pragya Shrestha ${ }^{1,2}$, Gon Namkoong ${ }^{1,2}$, Xianyu $\mathrm{Ao}^{3}$, \\ and Frank Müller ${ }^{3}$ \\ ${ }^{1}$ Department of Electrical Engineering, Old Dominion University, Norfolk, Virginia 23529, USA \\ ${ }^{2}$ The Applied Research Center, Newport News, Virginia 23606, USA \\ ${ }^{3}$ Max Planck Institute of Microstructure Physics, Halle, Germany \\ Received: 29 September 2010 / Revised: 26 October 2010 / Accepted: 26 October 2010 \\ C The Author(s) 2010. This article is published with open access at Springerlink.com
}

\begin{abstract}
Lithographically defined microporous templates in conjunction with the atomic layer deposition (ALD) technique enable remarkable control of complex novel nested nanotube structures. So far three-dimensional control of physical process parameters has not been fully realized with high precision resolution, and requires optimization in order to achieve a wider range of potential applications. Furthermore, the combination of composite insulating oxide layers alternating with semiconducting layers and metals can provide various types of novel applications and eventually provide unique and advanced levels of multifunctional nanoscale devices. Semiconducting $\mathrm{TiO}_{2}$ nanotubes have potential applications in photovoltaic devices. The combination of nanostructured semiconducting materials with nested metal nanotubes has the potential to produce novel multifunctional vertically-ordered three-dimensional nanodevices. Platinum growth by ALD has been explored, covering the initial stages of the thin film nucleation process and the synthesis of high aspect ratio nanotube structures. The penetration depth of the Pt into porous templates having various pore sizes and aspect ratios has been investigated. Several multi-walled nested $\mathrm{TiO}_{2}-\mathrm{Pt}$ nanotubes in series have been successfully fabricated using microporous Si templates. These innovative nested nanostructures have the potential to produce novel multifunctional vertically-ordered three-dimensional nanodevices in photovoltaic and sensing technologies.
\end{abstract}

\section{KEYWORDS}

Atomic layer deposition (ALD) platinum nanotubes, semiconductor/metal nanotubes, microporous Si templates, nanoporous alumina templates, multilayer nested nanotubes

For several decades, the fabrication of nanostructures having highly-ordered, complex architectures has been one of the most interesting research topics. These structures have the potential to advance current technologies and enable the development of newer and more innovative applications of all kinds. In the past few years, vertically-ordered, self-organized nanotubes and nanorods have been synthesized using anodic aluminum oxide (AAO) or silicon (Si) nano/micropore templates. Most coaxial nanotubes currently produced with nanotemplates maintain three-dimensional scales close to those of the original

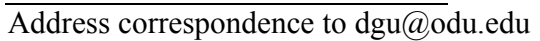


templates. Therefore, even though coaxial, nanostructured oxides such as $\mathrm{ZnO}, \mathrm{TiO}_{2}$, and other metal oxide mixtures have been demonstrated using nanotemplates [1], three-dimensional control of physical parameters has not been fully realized with highly precise resolution and needs to be optimized in order to achieve a wider range of potential applications. Furthermore, the combination of insulating oxides, semiconducting materials, and metals can provide the genesis for various types of novel applications and eventually provide unique and advanced levels of multifunctional nanoscale devices [2]. Recently, metal nanotubes employing platinum $(\mathrm{Pt})$ have been attracting intensive interest due to their excellent electrical properties, chemical stability, and suitability for many applications in microelectronics and catalysis. For example, platinum can be used as an electrode at high temperatures in both oxidative and reductive environments [3]. Because of its high work function, platinum is also appropriate for use as a gate metal with high- $\kappa$ dielectrics in metal-oxide-semiconductor field effect transistors (MOSFETs) [4, 5]. Additionally, $\mathrm{Pt}$ is widely used in fuel cells due to its high catalytic activity $[6,7]$. Therefore, the combination of nanostructured semiconducting materials and metal nanotubes has the potential to produce novel multifunctional vertically-ordered three-dimensional nanodevices. In this paper, we describe the fabrication of highly-ordered, multi-walled nested coaxial nanotube arrays composed of metal oxides and metal nanotubes based on precise control of threedimensional structural parameters, diameter, thickness, and nanotube length.

The realization of well-ordered electrodes is of critical importance in nanotechnology and can be achieved using atomic layer deposition (ALD) inside nano/micropore templates. Our main strategy is to utilize template-guided deposition by ALD to produce multi-walled nested coaxial nanotube architectures inside nano/micropore templates. The template-guided ALD technique allows for remarkable control of nested nanotube spacing within atomic resolution by simply adjusting the thickness of concentric sacrificial spacer layers. This concept was further used as a reproducible route to design and locate the different combinations of materials inside templates. In such a way, three-dimensional parameters including diameter, height, and thickness can be controlled with higher precision resolution and high aspect ratio.

Even though growth of metal oxide nanotubes such as $\mathrm{ZnO}$ and $\mathrm{TiO}_{2}$ has already been demonstrated [8], a detailed understanding of the mechanisms characteristic of metal nanotubes is still missing. Therefore, we first explored the formation of ALDgenerated $\mathrm{Pt}$ covering the initial stages of the thin film nucleation process and the synthesis of high aspect ratio nanotube structures developed using rimethyl(methylcyclopentadienyl)platinum(IV), $\mathrm{Me}_{3} \mathrm{Pt}$ (CpMe), and oxygen as the precursors [9-12]. The penetration depth of the $\mathrm{Pt}$ into porous templates having various pore sizes and aspect ratios was investigated using the template replication method.

$\mathrm{Me}_{3} \mathrm{Pt}(\mathrm{CpMe})$ (99\%) and high purity oxygen (99.9995\%) were purchased from Strem Chemicals, Inc., and GTS-Welco, respectively. The ALD-generated $\mathrm{Pt}$ thin films were initially grown on $\mathrm{Si}$ substrates so that film growth mechanisms and electrical properties could be analyzed. The pulse times for $\mathrm{Me}_{3} \mathrm{Pt}(\mathrm{CpMe})$ and oxygen were $1 \mathrm{~s}$ and $0.1 \mathrm{~s}$, respectively, separated by $5 \mathrm{~s}$ of purging pump time. The $\mathrm{Me}_{3} \mathrm{Pt}(\mathrm{CpMe})$ was heated to $80^{\circ} \mathrm{C}$ to achieve the required vapor pressure. The deposition temperature ranged from $260{ }^{\circ} \mathrm{C}$ to $340{ }^{\circ} \mathrm{C}$ in order to accommodate the ALD process window. In order to achieve a high deposition rate, a smooth surface topography and to minimize impurity content, a deposition temperature of $300{ }^{\circ} \mathrm{C}$ was determined to be optimum for the ALD process. The increase in growth rate at deposition temperatures higher than $320^{\circ} \mathrm{C}$ is indicative of the decomposition of the Pt precursor [7]. Figure 1(a) shows a crosssectional transmission electron microscopy (TEM, XTEM, JEOL 2100F) analysis of the initial stage of ALD Pt deposition on planar Si substrates covered with native oxide. At 50 ALD deposition cycles, no continuous $\mathrm{Pt}$ thin film was formed. Instead, only isolated Pt nanoparticles with sizes of less than $5 \mathrm{~nm}$ were found randomly distributed on the wafer. With an increase in number of ALD cycles, the Pt nanoparticles continued to grow in size and eventually the $\mathrm{Pt}$ nanoislands coalesced into larger islands. However, the Pt islands still remained isolated from each other as indicated in Fig. 1(b). Finally, as illustrated 
in Fig. 1(c), at 200 ALD cycles corresponding to a Pt thickness of approximately $10 \mathrm{~nm}$, the Pt islands coalesced further to form a connected Pt network corresponding to a discontinuous film with gaps. When the ALD growth exceeded 200 cycles, the Pt started to form a continuous thin film with a smooth surface as shown in Figs. 1(d) and 1(e) [13]. These TEM cross-sectional images display surface morphologies similar to those reported by Jiang et al. [12]. Once the substrate surface was completely covered with the coalesced Pt islands, the ALD film growth followed the classical ALD model of monolayer-by-monolayer growth per deposition cycle [14]. X-ray diffraction (XRD) shows a preferred (111) grain orientation and overall good quality polycrystalline ALD Pt films as depicted in the XRD pattern in Fig. 1(f). The various identified growth mechanisms of $\mathrm{Pt}$ can be utilized advantageously for technological purposes to control its deposition in various forms including metal nanoparticles, porous-discontinuous, and continuous Pt films. The resistivity of a $20 \mathrm{~nm}$ thick Pt film was measured by four point probe to be $12 \mu \Omega \cdot \mathrm{cm}$, which is very close to the bulk resistivity of $\mathrm{Pt}(10.8 \mu \Omega \cdot \mathrm{cm})$. This is attributed to the fact that a Pt film of $15 \mathrm{~nm}$ is sufficiently thick to achieve good electrical conductivity.

After the ALD optimum process for Pt was established, we studied conformal coating of high aspect ratio template structures. We explored the synthesis of $\mathrm{Pt}$ tubes on porous $\mathrm{AAO}$ and microporous $\mathrm{Si}$ templates [8,15-17]. The porous AAO templates have relatively small pore size with upper limits in the range $250-300 \mathrm{~nm}$ and a thickness of $60 \mu \mathrm{m}$. In contrast, microporous $\mathrm{Si}$ templates can attain much larger pore sizes and can be fabricated with pore diameters ranging from $500 \mathrm{~nm}$ to $4 \mu \mathrm{m}[18,19]$. For this study we have worked with $\mathrm{Si}$ templates having a pore size of $1 \mu \mathrm{m}$ and a pore depth of $90 \mu \mathrm{m}$. In the formation of $\mathrm{Pt}$ nanotubes, we found that the length of the nanotubes is primarily dependent upon the nano/micropore size and the precursor diffusion rate. The scanning electron microscope (SEM, JEOL JSM 6060 LV) cross-section views and energy dispersive $\mathrm{X}$-ray spectroscopy (EDS) mapping revealed that $\mathrm{Pt}$ can be deposited via ALD up to a depth of $10 \mu \mathrm{m}$ inside the AAO nanopore structure with no added $\mathrm{Pt}$ precursor exposure time. However, there exists the possibility that a Pt film thickness gradient will develop ranging from the surface into the pores. The

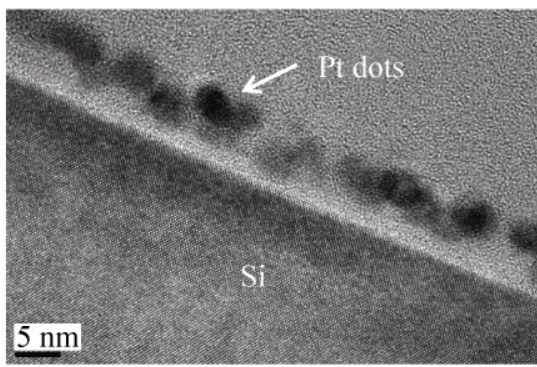

(a)

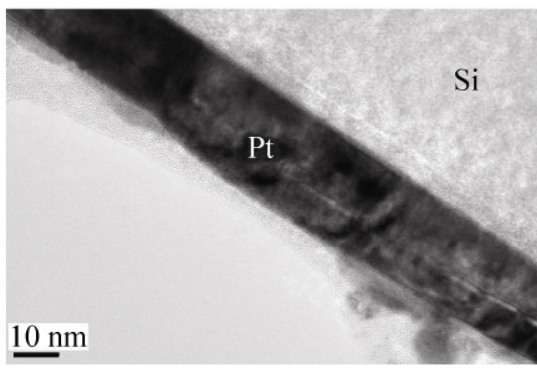

(d)

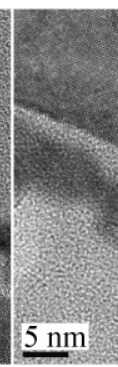

5 nm

(e)

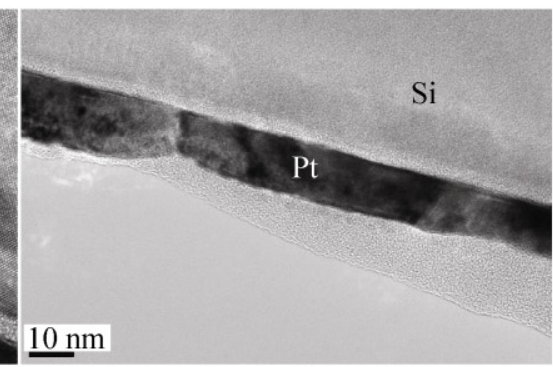

(c)

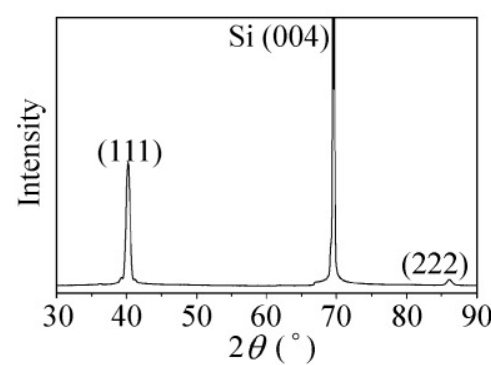

(f)

Figure 1 Cross-sectional TEM micrographs analyzing the deposition of Pt thin films by ALD at $300{ }^{\circ} \mathrm{C}$ for different thickness stages: (a) 50 ALD cycles, (b) 100 ALD cycles, (c) 200 ALD cycles, (d) 400 ALD cycles, (e) 1000 ALD cycles, and (f) XRD pattern of a 200 ALD cycle platinum film 
penetration of the ALD Pt coating into the nanoporous AAO template proceeded deeper when a $10 \mathrm{~s}$ exposure time was added for both the Pt precursor and oxygen. However, the Pt penetration depth into the nanoporous template saturated at around $20 \mu \mathrm{m}$ from the surface even with extended exposure times of $30 \mathrm{~s}$. Beyond this point the penetration depth of the Pt precursors into the AAO membrane did not increase any further as the exposure time was increased. This can be attributed to the combined effects of the very limited AAO pore size and the $\mathrm{Pt}$ precursor diffusion rate, which is inversely proportional to the square root of the molecular mass for this precursor [20]. Platinum nanotubes were synthesized in order to further analyze the quality of the thickness conformity of the Pt coating. This was accomplished by dissolving the AAO template in sodium hydroxide $(\mathrm{NaOH})$ solution. Figure 2 shows the individual nanotube structures that have been separated from each other after chemical dissolution of the porous AAO template.

Figure 2 reveals that the nanotubes are about $15 \mu \mathrm{m}$ in length. This length is less than the Pt penetration depth with a $30 \mathrm{~s}$ exposure time added. This finding can be explained by a tapering off in the ALD Pt coating thickness as a function of penetration depth, which is documented in the TEM inset images. These TEM micrographs were taken after the separation of the released $\mathrm{Pt}$ nanotubes. At one end of the nanotubes close to the template surface, the $\mathrm{Pt}$ film constitutes a continuous coating and forms dense $\mathrm{Pt}$ nanotubes. However, at the opposite end of the nanotube which is located deeper into the porous AAO template, the ALD Pt film coating is still discontinuous and forms porous $\mathrm{Pt}$ nanotubes (see the lower TEM inset in Fig. 2). Our analysis also confirms that only isolated $\mathrm{Pt}$ islands were formed for depths exceeding $15 \mu \mathrm{m}$ inside the nanoporous alumina template, which mirrors the initial VolmerWeber island growth observed on ALD Pt films on flat substrates.

Next we investigated the application potential of microporous $\mathrm{Si}$ templates with larger pore diameters in order to further increase nanotube length. The SEM images in Figs. 3(a)-3(c) exhibit the structure of the microporous $\mathrm{Si}$ template. The microporous $\mathrm{Si}$

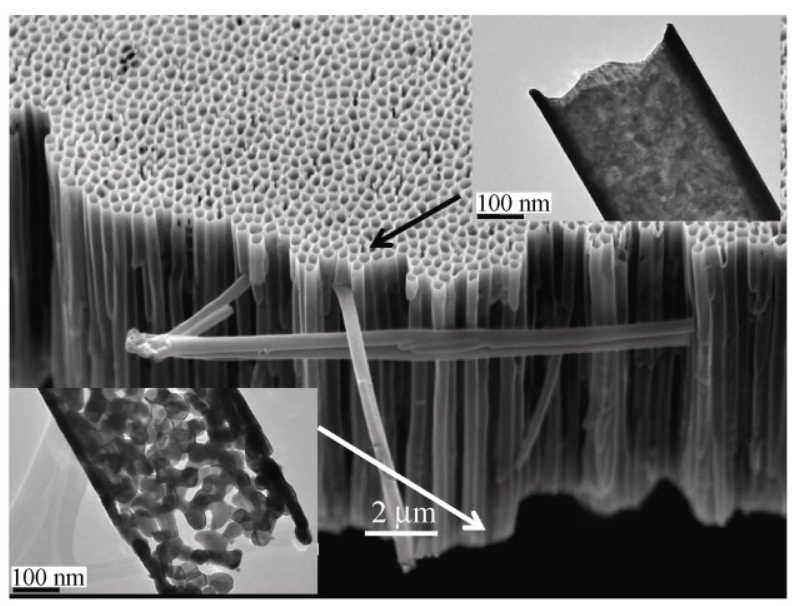

Figure 2 SEM micrograph of ALD platinum nanotubes fabricated using a $30 \mathrm{~s}$ exposure time for the Pt precursor. The insets at the upper right and lower left are TEM images for the Pt tube structures revealing a continuous solid Pt nanotube near the AAO template surface, and a discontinuous Pt nanotube with large gaps at the other end of the Pt nanotube deep inside the porous AAO template

templates are photolithographically defined in an ordered regular array of slightly square pores with a pore size of $1 \mu \mathrm{m}$, pore depth of $90 \mu \mathrm{m}$, and are $2 \mu \mathrm{m}$ apart. The uniform pore size from the top to the bottom is confirmed in cleaved cross-section SEM and shown in Fig. 3(c). After coating the inner walls of the porous Si template with Pt via ALD, etching was performed to release the nanotubes. Figure 3(d) shows the partially-released Pt nanotubes protruding from the surface after limited etching recessed the $\mathrm{Si}$ template by about $2 \mu \mathrm{m}$. The SEM image in Fig. 3(e) demonstrates that $\mathrm{Pt}$ was deposited to a depth of $30 \mu \mathrm{m}$ without any added ALD precursor exposure time. Comparing the different pore sizes of $1 \mu \mathrm{m}$ for the Si template, versus $\sim 300 \mathrm{~nm}$ for the AAO template, and correlating the $\mathrm{Pt}$ deposition depth, it is apparent that no advantage in aspect ratio was observed for ALD Pt deposition without added exposure time. However, the SEM image in Fig. 3(f) confirms that Pt was coated at the bottom of the porous Si template to a depth of $90 \mu \mathrm{m}$ when we used $10 \mathrm{~s}$ additional ALD exposure time. The thickness of the Pt coating might not be consistent from the top to the bottom of the Pt nanotube, but there is an advantage to using a microporous Si template for the ALD synthesis of much larger Pt nanotubes. In contrast to the nanoporous

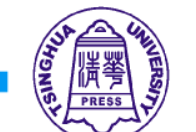

黑 Springer 


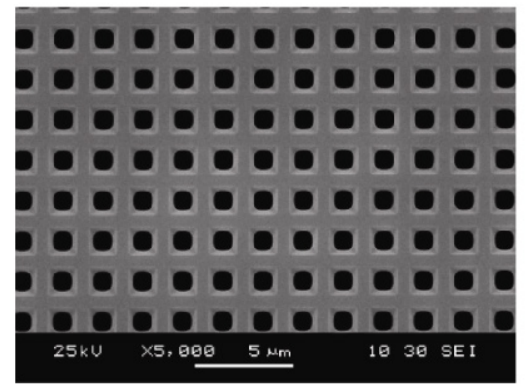

(a)

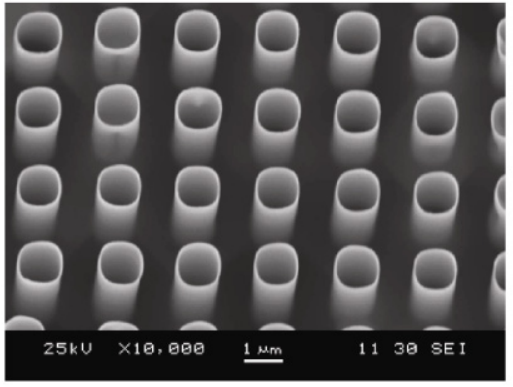

(d)

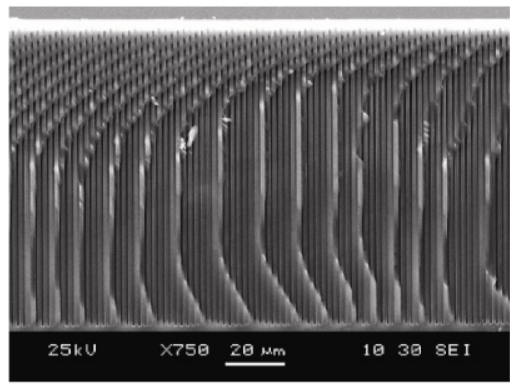

(b)

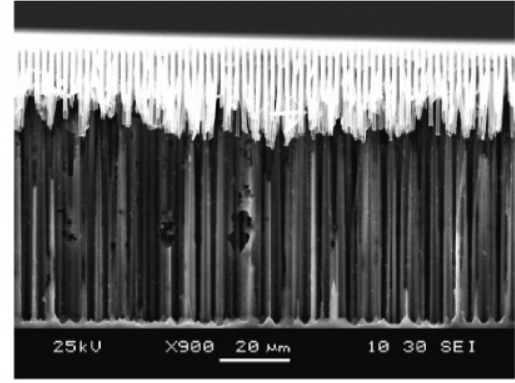

(e)

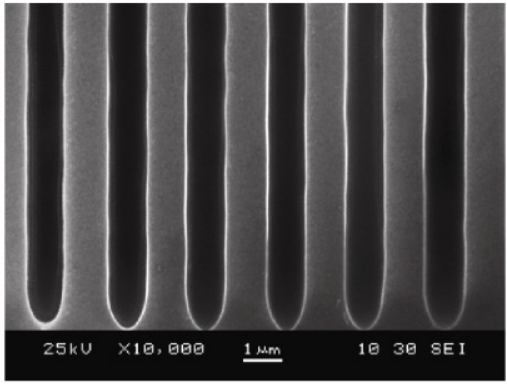

(c)

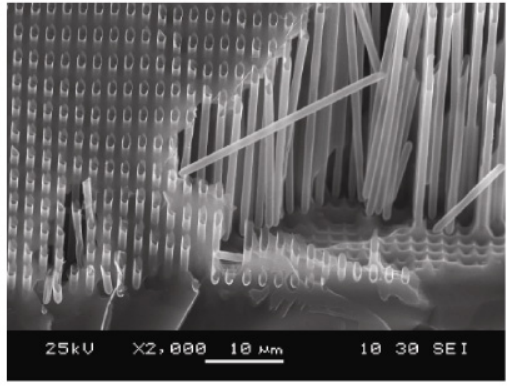

(f)

Figure 3 (a) SEM top view micrograph of the porous Si template with a pore size of $1 \mu \mathrm{m}$, (b) cross-sectional SEM view revealing the Si template pore depth of $90 \mu \mathrm{m}$, (c) SEM cross-section depicting uniformity of the radius of the Si pores, (d) partially released ALD Pt tubes protruding above etched and recessed surface of the microporous Si template, (e) cleavage site demonstrating that ALD Pt coating can only be deposited into the porous Si template to a penetration depth of $30 \mu \mathrm{m}$ with no added ALD exposure time, and (f) cleaved Si template highlighting that ALD Pt has infiltrated up to $90 \mu \mathrm{m}$ deep into the bottom of the porous Si template by using an additional $10 \mathrm{~s}$ ALD exposure time

AAO template, ALD Pt can be deposited deeper into microporous $\mathrm{Si}$ templates. This can be attributed to the larger pore size which facilitates the diffusion of the Pt precursor.

The advantages of microporous Si templates over AAO templates are the regularity in pore arrangement and the considerably larger pore size. The larger pore diameter enables fabrication of more complicated nested nanostructures that result from combining semiconducting materials and metal nanotubes with fully controlled three-dimensional parameters. The SEM micrographs in Fig. 4 show an advanced multilayered nested nanotube structure within the pores of the $\mathrm{Si}$ template composed of a sequence of $\mathrm{TiO}_{2}$ and $\mathrm{Pt}$ nanotubes (two in series), which has potential applications in photovoltaic devices [21]. $\mathrm{TiO}_{2}$ layers were grown with titanium isopropoxide ( $\left.\mathrm{Ti}\left({ }^{\mathrm{P} P \mathrm{PO}}\right)_{4}\right)$ and $\mathrm{H}_{2} \mathrm{O}$ at $250{ }^{\circ} \mathrm{C}$ [8]. $\mathrm{Al}_{2} \mathrm{O}_{3}$ layers were deposited using trimethylaluminum (TMA) and $\mathrm{H}_{2} \mathrm{O}$ at $300{ }^{\circ} \mathrm{C}$. The SEM image in Fig. 4(a) is a top view showing the sequence within the multilayer coaxial nested nanotube structure: $\mathrm{TiO}_{2}(50 \mathrm{~nm}) / \mathrm{Al}_{2} \mathrm{O}_{3}$ $(120 \mathrm{~nm}) / \mathrm{Pt}(25 \mathrm{~nm}) / \mathrm{TiO}_{2}(25 \mathrm{~nm}) / \mathrm{Al}_{2} \mathrm{O}_{3}(120 \mathrm{~nm}) / \mathrm{Pt}$ $(50 \mathrm{~nm}$ ) (in order from the outer pore walls towards the pore center). The compositions of the nested tube structure are denoted by the contrast and brightness. The $\mathrm{Pt}$ ring is brighter than $\mathrm{TiO}_{2}$ and $\mathrm{Al}_{2} \mathrm{O}_{3}$ because it has higher atomic mass. To achieve multilayered nested nanotubes separated by open annular sacrificial spacer layers, we used selective chemical etching methods. First, the $\mathrm{Al}_{2} \mathrm{O}_{3}$ sacrificial spacer layers and the porous $\mathrm{Si}$ template were partially removed by using $\mathrm{NaOH}$ solution. The SEM micrograph in Fig. 4(b) clearly delineates the partially released $\mathrm{TiO}_{2}, \mathrm{Pt} / \mathrm{TiO}_{2}$, and $\mathrm{Pt}$ nanotubes. Figure 4(c) depicts the etch depth, and long nanotubes at the edge of the Si substrate with the microporous Si template fully removed in $\mathrm{NaOH}$ solution from side etching. Figure 4(d) shows a fully released long entirely intact nanotube. It can be clearly seen that $\mathrm{TiO}_{2}, \mathrm{Pt} / \mathrm{TiO}_{2}$, and $\mathrm{Pt}$ nanotubes have been selectively etched and released from the $\mathrm{Al}_{2} \mathrm{O}_{3}$ sacrificial spacer layer and 


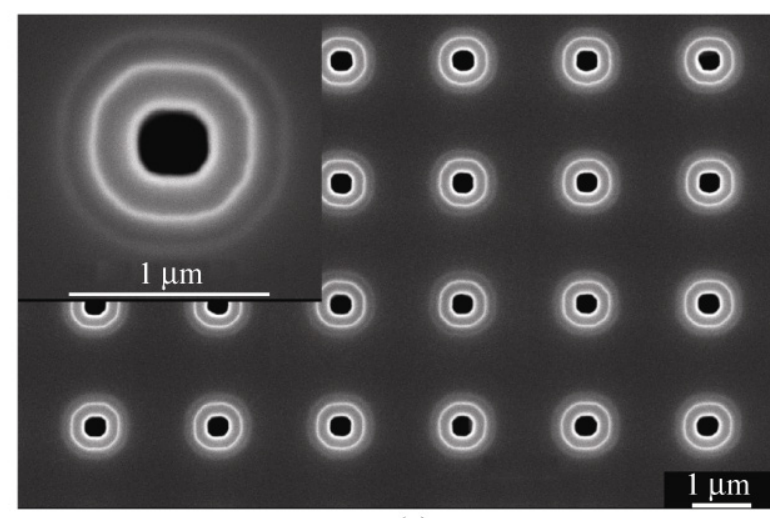

(a)

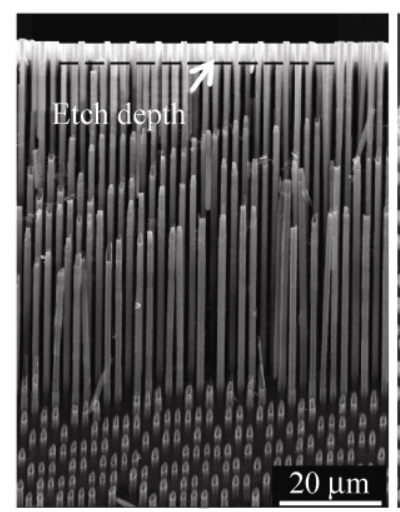

(c)

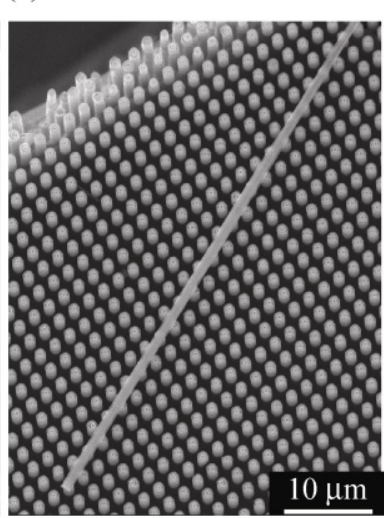

(d)

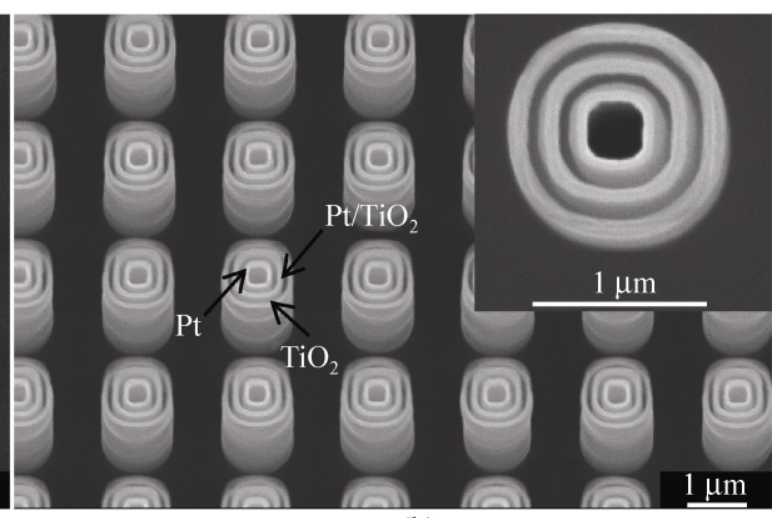

(b)

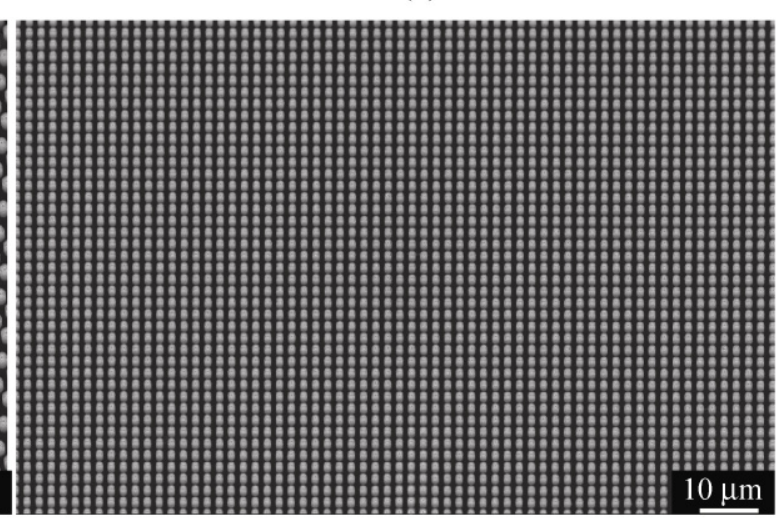

(e)

Figure 4 SEM micrographs revealing (a) multilayer nested composite nanotube structures synthesized by ALD inside a microporous $\mathrm{Si}$ template and composed of $50 \mathrm{~nm} \mathrm{TiO}_{2} / 120 \mathrm{~nm} \mathrm{Al} \mathrm{O}_{3} / 50 \mathrm{~nm} \mathrm{Pt} / 120 \mathrm{~nm} \mathrm{Al} \mathrm{O}_{3} / 50 \mathrm{~nm} \mathrm{Pt}$, starting from the pore wall towards the pore center, (b) multi-walled nested nanotube structures with alternating open annular layers after removal via chemical etching of two ALD $\mathrm{Al}_{2} \mathrm{O}_{3}$ sacrificial spacer layers, (c) etch depth from top and released long tubes from side, (d) an intact very long nanotube after etching and complete release from the Si template, and (e) the uniformity of an array of ALD nanotubes over a large area

microporous Si template. It should be noted that such nanotube arrays have highly-ordered nanostructures with a length of $\sim 90 \mu \mathrm{m}$ and an inter-nanotube distance of $120 \mathrm{~nm}$ yielding a high aspect ratio. Furthermore, Figs. 4(c) and 4(d) confirm that highly ordered nanotube arrays, possessing uniformity over a large area, can be achieved using microporous Si templates and ALD techniques.

In summary, we have shown how highly-ordered nanotube arrays may be fabricated over a large area using the ALD technique with template replication and selective chemical etching methods. Compared to existing coaxial techniques, our method provides more flexible design methodologies capable of producing composite nested nanotube materials containing semiconducting materials, metals, and insulating materials alternating with sacrificial spacer layers, which can also be formed through ALD. Furthermore, our improved method allows for precise control of three-dimensional parameters where currently-used conventional methods rely entirely upon the dimensions of nano/micropore templates. We have essentially identified a reproducible route to design and locate the different combinations of nested nanotube materials inside microporous templates. By modulating the ALD exposure time and the number of ALD growth cycles we can control all three-dimensional parameters of high aspect ratio nested nanotube structures including diameter, height, and thickness for verticallyordered nanodevices. Such top-down lithographically defined regular arrays of nested coaxial nanotubes combining numerous and physically diverse materials offer the promise of myriad novel applications including photocatalysis, sensors and detectors, bioengineering 
and biomedical applications, and photovoltaic devices Our method is capable of synthesizing custom designed metal nanotube structures that range from continuous intact ALD coatings and metal nanotubes to discontinuous metal film networks with large porosity and includes the special case of metal nanoparticles and quantum dots for catalytic applications and laser protection optics.

Open Access: This article is distributed under the terms of the Creative Commons Attribution Noncommercial License which permits any noncommercial use, distribution, and reproduction in any medium, provided the original author(s) and source are credited.

\section{References}

[1] Gu, D.; Baumgart, H.; Abdel-Fattah, T.; Namkoong, G. Atomic layer deposition of $\mathrm{ZrO}_{2}$ and $\mathrm{HfO}_{2}$ nanotubes by template replication. Electrochem. Solid-State Lett. 2009, 12, K25-K28.

[2] Herrmann, C. F.; Fabreguette, F. H.; Finch, D. S.; Geiss, R.; George, S. M. Multilayer and functional coatings on carbon nanotubes using atomic layer deposition. Appl. Phys. Lett. 2005, 87, 123110 .

[3] Hiratani, M.; Nabatame, T.; Matsui, Y.; Imagawa, K.; Kimura, S. Platinum film growth by chemical vapor deposition based on autocatalytic oxidative decomposition. J. Electrochem. Soc. 2001, 148, C524-C527.

[4] Wilk, G. D.; Wallace, R. M.; Anthony, J. M. Hafnium and zirconium silicates for advanced gate dielectrics. J. Appl. Phys. 2000, 87, 484-492.

[5] Gu, D.; Dey, S. K.; Majhi, P. Effective work function of Pt, $\mathrm{Pd}$, and $\mathrm{Re}$ on atomic layer deposited $\mathrm{HfO}_{2}$. Appl. Phys. Lett. 2006, 89, 082907.

[6] Jiang, X.; Huang, H.; Prinz, F. B.; Bent, S. F. Application of atomic layer deposition of platinum to solid oxide fuel cells. Chem. Mater. 2008, 20, 3897-3905.

[7] Liu, C.; Wang, C. C.; Kei, C. C.; Hsueh, Y. C.; Perng, T. P. Atomic layer deposition of Platinum nanoparticles on carbon nanotubes for application in proton-exchange membrane fuel cells. Small 2009, 5, 1535-1538.
[8] Gu, D.; Baumgart, H.; Abdel-Fattah, T. M.; Namkoong, G. Synthesis of nested coaxial multiple-walled nanotubes by atomic layer deposition. ACS Nano 2010, 4, 753-758.

[9] Aaltonen, T.; Ritala, M.; Sajavaara, T.; Keinonen, J.; Leskela, M. Atomic layer deposition of platinum thin films. Chem. Mater. 2003, 15, 1924-1928.

[10] Xue, Z.; Thridandam, H.; Kaesz, H. D.; Hicks, R. F. Organometallic chemical vapor-deposition of platinumreaction-kinetics and vapor-pressures of precursors. Chem. Mater. 1992, 4, 162-166.

[11] Hoover, R. R.; Tolmachev, Y. V. Electrochemical properties of Pt coatings on Ni prepared by atomic layer deposition. $J$. Electrochem. Soc. 2009, 156, A37-43.

[12] Jiang, X.; Bent, S. F. Area-selective atomic layer deposition of Platinum on YSZ substrates using microcontact printed SAMs. J. Electrochem. Soc. 2007, 154, D648-656.

[13] Puurunen, R. L. Surface chemistry of atomic layer deposition: A case study for the trimethylaluminum/water process. J. Appl. Phys. 2005, 97, 121301.

[14] Christensen, S. T.; Elam, J. W.; Lee, B.; Feng, Z.; Bedzyk, M. J.; Hersam, M. C. Nanoscale structure and morphology of atomic layer deposition platinum on $\mathrm{SrTiO}_{3}(001)$. Chem. Mater. 2009, 21, 516-521.

[15] Zhao, Y.; Guo, Y. G.; Zhang, Y. L.; Jiao, K. Fabrication and characterization of highly ordered Pt nanotubule arrays. Phys. Chem. Chem. Phys. 2004, 6, 1766-1768.

[16] Luo, Y.; Lee, S. K.; Hofmeister, H.; Steinhart, M.; Gösele, U. Pt nanoshell tubes by template wetting. Nano Lett. 2004, 4, 143-147.

[17] Liu, L.; Yoo, S. H.; Park, S. Synthesis of vertically aligned hollow platinum nanotubes with single crystalline nanoflakes. Chem. Mater. 2010, 22, 2681-2684.

[18] Lehmann, V. The physics of macropore formation in low doped n-type silicon. J. Electrochem. Soc. 1993, 140, 2836-2843.

[19] Matthias, S.; Müller, F.; Schilling, J.; Gösele, U. Pushing the limits of macroporous silicon etching. Appl. Phys. A. 2005, 80, 1391-1396.

[20] Scott, D. S.; Dullien, F. A. L. Diffusion of ideal gases in capillaries and porous solids. AIChE J. 1962, 8, 113-117.

[21] Foong, T. R. B.; Shen, Y.; Hu, X.; Sellinger, A. Templatedirected liquid ALD growth of $\mathrm{TiO}_{2}$ nanotube arrays: Properties and potential in photovoltaic devices. Adv. Funct. Mater. 2010, 20, 1390-1396. 